\title{
Abordaje Laparoscópico en el Testículo no Palpable
}

\author{
PEDRO-JOSÉ LÓPEZ E. ${ }^{1}$, LORENA ANGEL G. ${ }^{1}$, JORGE RODRÍGUEZ H. ${ }^{2}$, DANIELLE REYES C. ${ }^{2}$, \\ JOSÉ M. ESCALA A. ${ }^{1}$, MARÍA G. RETAMAL P. ${ }^{1}$, NELLY LETELIER C. ${ }^{1}$, RICARDO ZUBIETA A. ${ }^{1}$ \\ 1. Unidad Urología Pediátrica, Hospital Dr. Exequiel González Cortés, Santiago, Chile. \\ 2. Unidad de Urología Pediátrica, Hospital Luis Calvo Mackenna, Santiago, Chile.
}

\section{ABSTRACT \\ Laparoscopic Approach in the Management of Non-palpable Testes}

Objective: To evaluate our experience in laparoscopic management as the procedure of choice for nonpalpable testes (NPT). Methods: Review of charts of all boys with NPT who underwent laparoscopy (January 2000 December 2005). Demographic data, ultrasound (US) results, surgical details were documented. Results: Sixty-three patients (74 NPT) were identified; 25 left, 26 right and 12 bilateral. Mean age at diagnosis was 3.6y (1m-13y). Thirty-seven cases had a pre-op US; in 29 (78\%) the testis was not found; $7(22 \%)$ an intra-canalicular teste was described. All were impalpable at evaluation under anesthesia (EUA). Mean age at surgery was 4.9y ( 2 m-17 y). Laparoscopic findings were; intra-abdominal teste $(\mathrm{n}=24,33 \%)$, vanishing teste $(\mathrm{n}=12,16 \%)$ and vas-vessels entering inguinal ring $(\mathrm{n}=38,51 \%) .16 / 24$ underwent a 2 stage Fowler-Stephens orchyopexy and 8/24 a laparoscopic-assisted single stage orchypexy. When vasvessels entered inguinal ring, inguinal exploration was performed; 23 cases underwent orchyopexy; 13 had orchydectomy (atrophic-vanishing). After follow-up, 3 testes are atrophic. Conclusions: 36/74 NPT were not in the inguinal canal (24 intraabdominal and 12 vanishing). When vas-vessels entered inguinal ring, 13 were vanishing-atrophic and not identifiable (EUA or US). Hence, in 49 cases (66\%) laparoscopic exploration was critical in teste location, therefore this approach may be the procedure of choice for NPT.

(Key words: Impalpable, testes, laparoscopy, pediatric, intra-abdominal).

Rev Chil Pediatr 2009; 80 (3): 225-230

\section{RESUMEN}

Objetivo: Revisar nuestra experiencia en el abordaje laparoscópico como primer procedimiento en el estudio de teste no palpable (TNP). Método: Todas las fichas de niños con diagnóstico de TNP sometidos a laparoscopia entre Enero 2000 y Diciembre 2005 fueron revisadas. Se documentó detalles demográficos, ecográficos, laparoscópicos y de la cirugía realizada. Resultados: 63 pacientes con 74 TNP fueron identificados; todos fueron no palpados al examen bajo anestesia (EBA). Bajo visión laparoscópica, en 38/ $74(51 \%)$ el conducto deferente y los vasos se introducían en el orificio inguinal profundo, realizándose una

Trabajo recibido el 01 de diciembre de 2008, devuelto para corregir el 19 de enero de 2009, segunda versión el 04 de abril de 2009, aceptado para publicación el 27 de abril de 2009.

Correspondencia a:

Dra. Lorena Angel G.

E-mail: loreangelg@yahoo.es 
exploración inguinal abierta; 13/38 eran atróficos/evanescentes, en 23 se realizó orquidopexia, en 1 se realizó un primer tiempo de Fowler-Stephens y 1 no fue descrito. De los 36 (49\%) testes intra-abdominales, 12 eran evanescentes, en 8 se realizó una orquidopexia asistida por laparoscopia y los otros 16/36 se sometieron a un primer tiempo de F-S. En el post-operatorio hubo 3 testes atróficos; 2 luego de un segundo tiempo de F-S y 1 luego de una orquidopexia en primer tiempo fallida. Conclusiones: De los 74 TNP, 36 no estaban en el canal inguinal (24 intrabdominales y 12 atróficos/evanescentes). De los casos en que los vasos y deferente se dirigían al anillo inguinal $(\mathrm{n}=38), 13$ eran atróficos/evanescentes, lo que explicaría por qué no fueron detectados al EBA y/o ecografía. Por lo tanto, en 66\% (49/74) de los niños en esta serie, la exploración laparoscópica fue crítica en determinar la localización testicular, lo que la hace recomendable para el enfrentamiento del TNP.

(Palabras clave: Testículo no palpable, laparoscopia, pediatría, intra-abdominal).

Rev Chil Pediatr 2009; 80 (3): 225-230

\section{Introducción}

El escroto vacío es una de las causas más frecuentes de consulta en urología pediátrica. Dentro de este escenario, la criptorquidea es la causa más común, afectando a 1 de cada 150 niños. En $20 \%$ de estos pacientes, no se logra palpar el testículo, correspondiendo a teste no palpable (TNP).

A pesar de los avances en el diagnóstico y tratamiento de la criptorquidea, el manejo del teste no palpable continúa siendo un desafío. Si bien disponemos de varias pruebas que nos podrían ayudar en el diagnóstico (ecografía, TAC, RNM), ninguna ofrece el $100 \%$ de seguridad en determinar la localización o existencia del teste no palpable. Otra herramienta diagnóstica es la exploración inguinal, pero su principal problema es el posible daño a la irrigación, fundamental para realizar un $2^{\circ}$ tiempo de Fowler Stephens. Es aquí donde la laparoscopia tiene su rol fundamental.

El objetivo de este trabajo es revisar nuestra experiencia en el abordaje laparoscópico como primer procedimiento en el estudio de teste no palpable (TNP).

\section{Pacientes y Método}

Estudio descriptivo y retrospectivo. Se revisaron todas las fichas de niños con diagnóstico de testículo no palpable entre Enero 2000 y Diciembre 2005, a los que se les practicó una laparoscopia exploradora en 2 centros pediá- tricos; Hospital Dr. Exequiel González Cortés y Hospital Dr. Luis Calvo Mackenna.

El protocolo utilizado en esta revisión, consistió en examen clínico realizado por un urólogo pediátrico de experiencia. Si al examen físico no se encuentra teste en escroto, éste se realiza nuevamente bajo anestesia general. Si se mantiene el diagnóstico se realiza una laparoscopia exploradora que definirá la siguiente conducta. En caso de existir entrada de elementos nobles en orificio inguinal profundo se realiza un abordaje inguinal complementario. En caso de encontrar el teste en el abdomen la conducta a seguir depende de las características de este último: si es de características normales se realiza su descenso en uno o dos tiempos. Por otro lado, si el testículo es atrófico se realiza su extirpación y si es evanescente se concluye la intervención (figura 1).

Se documentó detalles demográficos, utilización o no de ecografía diagnóstica, hallazgos laparoscópicos, así como de la cirugía realizada.

Los resultados se presentan como medida de tendencia central y dispersión.

\section{Resultados}

Durante el período de cinco años estudiado, sesenta y tres pacientes con 74 TNP fueron sometidos a exploración laparoscópica; 25 izquierdos, 25 derechos y 12 bilaterales. El promedio de edad al momento del diagnóstico fue de 3,65 años, con rango entre 1 mes y 13 años. 


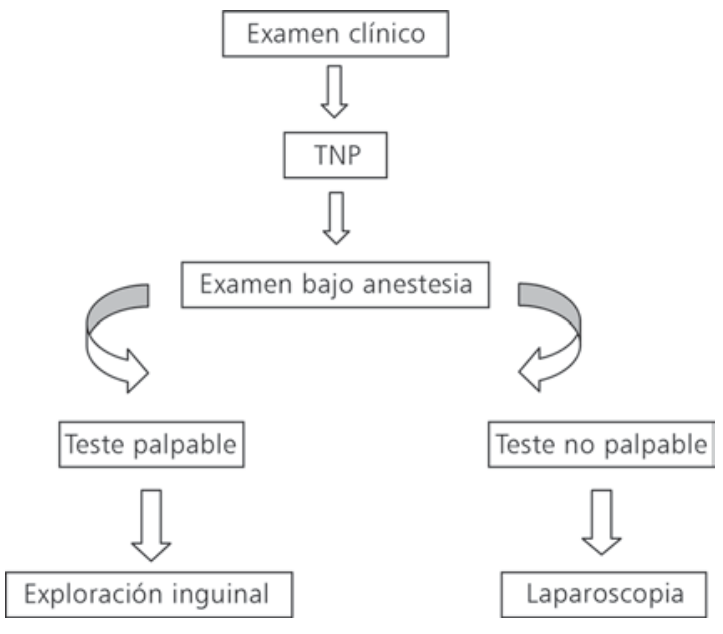

Figura 1. Protocolo Estudio TNP.

Treinta y siete casos (58\%) tuvieron una ecografía pre operatoria: en $29(78 \%)$ el teste no fue encontrado; en 7 el teste se identificó como intracanalicular y 1 fue identificado como intra escrotal. De los 29 pacientes en que la ecografía fue negativa, se encontró durante la cirugía que en $16(55 \%)$ el teste estaba intracanalicular (12 descritos como atróficos y 4 normales).

Todos los testes eran no palpables al examen bajo anestesia (EBA). El promedio de edad al momento de la cirugía fue 4,9 años (rango 2 meses-17 años), con un tiempo promedio operatorio de 75,5 minutos (rango 10 minutos-155 minutos).

Bajo visión laparoscópica, en 38 de 74 testes $(51 \%)$, el conducto deferente y los vasos se introducían en el orificio inguinal profundo, por lo tanto, se realizó una exploración inguinal abierta. De estos 38, 13 eran atróficos o evanescentes, en 23 se realizó orquidopexia, en 1 se realizó un primer tiempo de Fowler-Stephens y 1 no fue descrito. De los 36 testes intraabdominales, 12 eran evanescentes, en 8 se realizó una orquidopexia asistida por laparoscopia en un tiempo y los otros 16 se sometieron a un primer tiempo de Fowler-Stephens. El promedio de hospitalización en esta serie fue de $24 \mathrm{hrs}$.

Hubo $3(4,7 \%)$ pacientes que presentaron alguna complicación peri operatoria: infección de herida operatoria $(n=1)$, fiebre $(n=1)$, hematoma $(\mathrm{n}=1)$. En cuanto a las complicaciones tardías, hubo tres testes que evolucionaron en forma atrófica; 2 luego de un segundo tiempo de Fowler-Stephens y 1 luego de una orquidopexia en primer tiempo.

El promedio de seguimiento de los pacientes fue 3,3 controles, con un rango de 1 a 10 .

\section{Discusión}

El testículo no descendido es aquel que no se encuentra en el escroto ni puede ser llevado a él por medio de maniobras ${ }^{1}$. El primer estudio publicado respecto de este tema es de John Hunter, en 1786, quien encontró que en el abdomen de fetos de 7 meses había testículos y, en cambio, en los de 9 meses estos se encontraban en el escroto ${ }^{1}$. En $20 \%$ de estos pacientes, no se logra palpar el testículo, correspondiendo a lo que se denomina teste no palpable (TNP) $)^{1-4}$.

Un teste puede ser no palpable cuando está en el canal inguinal, en el abdomen o cuando es disgenético o evanescente ${ }^{1,3-7}$. La habilidad para diferenciar estas condiciones en el preoperatorio es lo más deseable y ventajoso ${ }^{1,2}$.

Nuestro objetivo como cirujanos es el de localizarlos y procurar su descenso, ya que el riesgo de degeneración del testículo no descendido se calcula del $2-6 \%$, siendo este riesgo mayor en el teste intra-abdominal $1^{1,3,6-9}$.

Hasta ahora no ha habido un método no invasivo de certeza para localizar el teste no palpable ${ }^{1}$. Existen, sin embargo, diferentes alternativas para su estudio que incluye el examen físico acucioso, incluso bajo anestesia, exámenes radiológicos, y procedimientos quirúrgi$\cos ^{4,6,7,9}$.

En cuanto al examen físico, su sensibilidad es dependiente de la experiencia del examinador, la morfología del paciente y la localización testicular ${ }^{4,6}$. El hallazgo de un teste descendido hipertrófico contralateral podría correlacionarse con ausencia de teste no palpable, pero este fenómeno bien documentado varía con la edad, el volumen total de tejido testicular presente y con la calidad del tejido descendido ${ }^{10}$.

Se ha descrito que de los pacientes con 
TNP, existe cerca de un $18 \%$ que en el EBA se logra palpar un testículo ${ }^{6}$. Esto destaca la importancia de re-examinar al paciente anestesiado previo a la intervención laparoscópica ${ }^{6}$. Lamentablemente no contamos con el registro de aquellos pacientes derivados con diagnóstico de TNP y que sí fueron palpados por nuestro equipo, siendo excluidos del protocolo, así como tampoco contamos con datos de aquellos niños que ingresaron a pabellón con diagnóstico de TNP, pero que al realizar el EBA se logró palpar el teste y no se realizó exploración laparoscópica.

Varias modalidades de imágenes diagnósticas han sido usadas para la investigación del teste no palpable, incluyendo la ecografía, la TC, la RNM y la RNM con gadolinio ${ }^{4,6,7,9}$. Sin embargo, ninguna de ellas puede predecir con exactitud la posición o morfología testicular, estimándose una exactitud de $44 \%$ para ellas ${ }^{7}$. Sin embargo, son de utilidad en aquellos casos en que el teste parece no palpable al examen clínico y se demuestra su posición intracanalicular por medio de alguna de ellas, por ejemplo con ecografía inguinal ${ }^{7}$. En estos pacientes se realiza una exploración inguinal y se previene la necesidad de laparoscopia.

La ecografía es de utilidad en la localización de un número significativo de teste intracanalicular, pero no es superior a un buen examen físico, excepto en pacientes obesos ${ }^{7}$. Se describe una sensibilidad de $97 \%$ para testes inguinales viables y de $48 \%$ para testes abdominales via$\mathrm{bles}^{7}$. En términos generales, inguinales y abdominales, se reporta una sensibilidad de $76 \%$ y una especificidad de $100 \%{ }^{7}$.

En esta serie la ecografía no formó parte del protocolo. Al realizarse dependiendo del médico, sólo 37 casos (58\%) tuvieron una ecografía pre operatoria: en $29(78 \%)$ el teste no fue encontrado; en 7 el teste se identificó como intracanalicular y 1 fue identificado como intra escrotal. Lamentablemente no existen detalles sobre que motivó al cirujano a realizar una laparoscopia con ecografía $(+)$.

De los 29 pacientes en que la ecografía fue negativa, en $16(55 \%)$ se identificó teste intracanalicular durante el procedimiento quirúrgico, lo que haría pensar en la baja sensibilidad de ésta. Sin embargo, 12/16 (75\%) son descritos como atróficos, lo que explicaría el hallazgo negativo ecográfico.

Otra herramienta diagnóstica es la exploración inguinal. En el caso de encontrar el teste en el canal inguinal, la conducta dependerá de las características de éste: si es de características normales se realiza su descenso, si el testículo es atrófico se realiza su extirpación y si es evanescente se concluye la intervención. Sin embargo, muchos de los cirujanos que realizan este procedimiento se han encontrado con el dilema de qué hacer cuando la exploración inguinal inicial no revela una gónada distal al anillo inguinal interno ${ }^{11}$. En tales circunstancias no se puede asegurar que el teste esté verdaderamente ausente o si la búsqueda fue inadecua$\mathrm{da}^{11}$. Además en estos casos el principal problema de la exploración inguinal es el posible daño a la irrigación local, fundamental para realizar un $2^{\circ}$ tiempo de Fowler Stephens. Otros autores describen a la exploración inguinal como un método poco fiable de investigación en el TNP, con una inaceptable alta incidencia de falso negativo o hallazgos inconclusos ${ }^{9}$. Es aquí donde la laparoscopia tiene su rol fundamental.

Ninguno de los exámenes previamente descritos (TAC, RNM, Ecografía) tiene la sensibilidad y especificidad que se logra con la laparoscopia $^{12}$. La laparoscopia provee de visualización directa de vasos testiculares y localiza el teste intra-abdominal, incluso aquellos evanescentes. Es de gran importancia tanto para el diagnóstico como para el tratamiento del TNP $\mathrm{TN}^{1,6}$. Cortesi describe por primera vez en 1976 la utilidad de la laparoscopia con fines diagnósticos, pero su verdadero desarrollo ocurrió en la década de los $90^{2,4,6,8}$. A partir de entonces su uso se amplió, constituyendo un estándar de referencia para la identificación y localización testicular, con una seguridad de $97 \%{ }^{6}$.

En general debemos pensar que en aproximadamente la mitad de los casos se va a encontrar un testículo adecuado ${ }^{8,9}$. Estos testes se descienden con diferentes técnicas como orquidopexia inguinal, orquidopexia ayudada de laparoscopia, orquidopexia realizada enteramente por vía laparoscópica y por la técnica de Fowler-Stephens en aquellos casos más difíciles con actuación en un tiempo o bien en 2 tiempos $^{6-9}$. Igualmente en cerca de la mitad de 
los casos la gónada no se va a encontrar, o va a ser de características hipoplásicas, requiriendo su extirpación, ya sea por vía laparoscópica o inguinal ${ }^{8,9}$.

En nuestra serie se identificó 38/74 testes en que el conducto deferente y los vasos se introducían en el orificio inguinal profundo, por lo tanto, se realizó una exploración inguinal abierta. Trece/38 eran atróficos o evanescentes, lo que podría explicar porque no fueron detectados al EBA y/o ecografía. En 23 testes se realizó orquidopexia (3 de ellos atróficos) y en 1 un primer tiempo de Fowler-Stephens.

Los 36 testes restantes eran intra-abdominales (12 evanescentes), permitiendo que en $24 / 36$ se realizara un procedimiento terapéutico asociado; en 8/24 se realizó una orquidopexia asistida por laparoscopia en un tiempo y los otros 16/24 se sometieron a un primer tiempo de Fowler-Stephens. De estos últimos, 2 evolucionaron con atrofia testicular, complicación descrita en este procedimiento.

Como se trata de un trabajo retrospectivo, de dos centros distintos, no existe registro fidedigno de lo que motivó los distintos enfrentamientos antes descritos. Se entiende que cada decisión correspondió al cirujano a cargo de cada procedimiento, lamentablemente sin corresponder a un protocolo establecido.

Snodgrass plantea que la incisión inicial en TNP unilateral debe ser la vía escrotal. Refiere que esta exploración podría ser definitiva cuando un teste atrófico es identificado ${ }^{13}$. Si en los pacientes de dicha serie se hubiese realizado la exploración laparoscópica como examen inicial, en el $60 \%{ }^{24}$ de ellos se hubiese evitado otra incisión para su diagnóstico. Además existe preocupación de que la estructura encontrada en escroto pueda corresponder a un segmento de deferente o epidídimo largo, con un correspondiente teste intra-abdominal ${ }^{13}$.

Finalmente, nuestra alternativa de manejo en TNP luego de esta revisión se aprecia en la figura 2. Todo TNP debe ser examinado por un urólogo pediátrico de experiencia. Si al examen físico no se palpa teste, se realiza una ecografía inguinal. Si ésta evidencia teste en el canal inguinal, el paciente va a exploración inguinal. Si la ecografía es negativa, se realiza nuevamente el examen clínico bajo anestesia general.

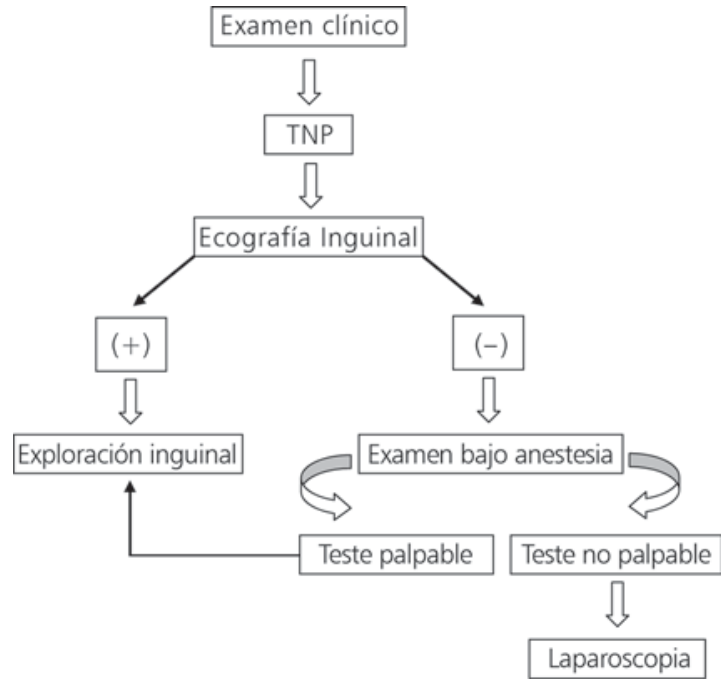

Figura 2. Protocolo Actual Estudio TNP.

Si se mantiene el diagnóstico se realiza una laparoscopia exploradora que definirá la siguiente conducta según lo analizado previamente.

\section{Conclusiones}

De los 74 TNP, 36 no estaban en el canal inguinal (24 intra-abdominales y 12 atróficos/ evanescentes), para los cuales la laparoscopia fue fundamental. En los casos en que los vasos y deferente se dirigían al anillo inguinal $(n=38)$, 13 eran atróficos/evanescentes, lo que explicaría porque no fueron detectados al EBA y/o ecografía. Por lo tanto, si evaluamos ambos grupos en conjunto, en 66\% (49/74) de los niños en esta serie, la exploración laparoscópica fue crítica en determinar la localización testicular. Los autores sugieren que el abordaje laparoscópico siguiría siendo el primer procedimiento para enfrentar un TNP.

\section{Referencias}

1.- Rostión CG: Testículo no descendido. Rev Chil Pediatr 2000; 71: 3 .

2.- Escala JM, Smith G, Zinder H, Duckett J: Testículo no palpable, características y tratamiento. Rev Chil Pediatr 1998; 69 (2): 68-71. 
3.- Wee Ang C, Forrest J: Diagnostic laparoscopy and management of the impalpable testis - a review of 10 years` practice at a non-paediatric specialist centre. Journal of Pediatric Urology 2008; 4: 214-7.

4.- Nijs S, Eijsbouts $S$, Madern G, Leyman P, Lequin M, Hazebroek F: Nonpalpable testes: is there a relationship between ultrasonographic and operative findings?. Pediatr Radiol 2007; 37: 374-9.

5.- Gulanikar AC, Anderson PAM, Schwarz R, Giacomantonio $M$ : Impact of diagnostic laparoscopy in the management of the unilateral impalpable testis. British Journal of Urology 1996; 77: 455-7.

6.- Patil K, Green J, Duffy P: Laparoscopy for impalpable testes. BJU International. 2005; 95: 704-8.

7.- Hutson J, Clarke M: Current management of the undescended testicle. Seminars in Pediatric Surgery 2007; 16: 64-70.

8.- De Mingo L, Vila JJ, Garrido M, Broto J, Beltrá R: El testículo no palpable y su tratamiento por laparoscopia. Revisión conjunta del Grupo Español de
Endocirugía Pediátrica (GEEP). Cir Pediatr 2003; 16: 139-41.

9.- Lakhoo K, Thomas DFM, Najmaldin AS: Is inguinal exploration for the impalpable testis an outdated operation?. British Journal of Urology 1996; 77: 4524.

10.- Mesrobian GO, Chassaignac JM, Laud PW: The presence or absence of an impalpable testis can be predicted from clinical observations alone. BJU International 2002; 90: 97-9.

11.- Barqawi A, Blyth B, Jordan G, Ehrlich R, Koyle M: Role of laparoscopy in patients with previous negative exploration for impalpable testis. Urology 2003; 61: 1234-7.

12.- Kaplan $G W$ : The undescended testis: changes over the past several decades. BJU International 2003; 92, Supplement 1: 12-142.

13.- Snodgrass $W$, Chen $K$, Harrison $C$ : Initial scrotal incision for unilateral nonpalpable testis. The Journal of Urology 2004; 172: 1742-5. 